\title{
Prevotella intermedia y enfermedad periodontal en embarazadas
}

\section{Prevotella intermedia and periodontal disease in pregnant women}

\author{
Guido Sebastián Falcón-Pasapera $^{1 \mathrm{a}}$, Britto Ebert Falcón-Guerrero ${ }^{2 b}$ \\ ID https://orcid.org/0000-0002-9585-7052 \\ (iD) https://orcid.org/0000-0003-3433-1599 \\ ${ }^{1}$ Universidad Peruana Cayetano Heredia, Facultad de Odontología. Lima, Perú. \\ ${ }^{2}$ Universidad Latinoamericana CIMA. Tacna, Perú. \\ ${ }^{a}$ Estudiante. \\ ${ }^{\mathrm{b}}$ Doctor en Estomatología. Especialista en Periodoncia e implantología. Profesor.
}

\begin{abstract}
Resumen
La enfermedad periodontal causa la pérdida de dientes en las mujeres embarazadas. Existe mucha evidencia que relaciona al embarazo con el desarrollo de las enfermedades periodontales, debido a la relación entre las hormonas sexuales con los tejidos periodontales; sin embargo, lo que llama la atención es la afinidad que hay entre estas hormonas con la prevalencia de la bacteria Prevotella intermedia. En tal sentido, se realizó una revisión bibliográfica actualizada con el objetivo de analizar si existe relación entre la presencia de la Prevotella intermedia con el desarrollo y la magnificación de las enfermedades periodontales en las mujeres embarazadas. Tomando en cuenta la evidencia científica analizada, se llegó a la conclusión que sí hay relación entre la Prevotella intermedia y el desarrollo y magnificación de las enfermedades periodontales durante la etapa del embarazo. Por lo cual, es siempre recomendable realizar la etapa preventiva y no quirúrgica del tratamiento.
\end{abstract}

Palabras clave: Periodontitis, prevotella intermedia, enfermedad periodontal, embarazo. (DeCS)

\begin{abstract}
Periodontal disease causes tooth loss in pregnant women. There is a lot of evidence linking pregnancy with the development of periodontal diseases, due to the relationship between sex hormones with periodontal tissues; however, what is striking is the affinity between these hormones with the prevalence of the bacteria Prevotella intermedia. In this sense, an updated bibliographic review was carried out, with the aim of analyzing whether there is a relationship between the presence of the Prevotella intermedia with the development and magnification of periodontal diseases in pregnant women. Taking into account the scientific evidence analyzed, it was concluded that there is a relationship between Prevotella intermedia and the development and magnification of periodontal diseases during the pregnancy stage. Therefore, it is always advisable to carry out the preventive and non-surgical stage of treatment.
\end{abstract}

Keywords: Periodontitis, Prevotella intermedia, Periodontal Disease, Pregnancy.

\section{Introducción}

La cavidad oral está colonizada por más de 700 especies microbianas que forman un ecosistema dinámico y complejo; estableciendo una simbiosis con el huésped, que puede variar dependiendo de la dieta, el estilo de vida y su estado de salud. Existe equilibrio entre la coexistencia de las poblaciones microbianas en los epitelios orales que promueven la protección de los tejidos contra los xenobióticos y las interacciones perjudiciales para el huésped. En caso de relaciones disbióticas, los miembros de la microbiota pueden activar las células inmunes provocando enfermedades orales inflamatorias crónicas (1).

Una de estas enfermedades es la enfermedad periodontal (EP), que es un proceso inflamatorio que afecta las estructuras de soporte de los dientes; lo que 
provoca la pérdida de los dientes y contribuye a la inflamación sistémica. El inicio y la propagación de la EP se da a través de una disbiosis de la microbiota oral comensal (placa dental), debido a que interactúa con las defensas inmunes del huésped, lo que provoca inflamación y enfermedad. La gravedad de la EP depende de factores ambientales y factores de riesgo del huésped; tanto modificables (fumar) como no modificables (susceptibilidad genética) (2).

Socransky et al. (3) propusieron seis complejos o "clusters" microbianos que llevan al desarrollo de la inflamación gingival. Los complejos amarillos (especies de Streptococcus), verdes (Campylobacter concisus, Eikenella corrodens), púrpuras (Veillonella parvula) y azules (especies de Actinomyces) están asociados con la salud periodontal; mientras que los complejos rojos (Porphyromonas gingivalis, Treponema denticola, Tannerella forsythia) y naranja (Fusobacterium, Prevotella y Campylobacter) son los principales patógenos periodontales asociados con la enfermedad.

Aunque los miembros centrales de los complejos rojos (particularmente $P$. gingivalis) se consideran "patógenos clave", se requiere la colonización subgingival por especies del complejo naranja (Prevotella intermedia) para el establecimiento de las especies del complejo rojo (4).

Fuera de la carga bacteriana se requiere que exista interacción entre factores ambientales, microbianos y los dependientes de la respuesta del huésped; dentro de los que figura el embarazo, donde se dan cambios hormonales que alteran los tejidos periodontales ante la presencia de agentes irritantes como la placa bacteriana (5); dado que las embarazadas presentan cambios fisiológicos que favorecen la colonización y la adherencia del complejo rojo y de la Prevotella intermedia, así como microorganismos asociados con la gingivitis y la EP crónica (6).

Basados en estos antecedentes, se tiene como objetivo realizar una búsqueda bibliográfica actualizada para determinar si la Prevotella intermedia tiene relación con el desarrollo de la EP, que afecta a una paciente embarazada.

\section{Prevotella intermedia}

Muchas especies del género Prevotella son patógenos que causan enfermedades orales; como la EP, periodontitis periapical y noma (7). Estas especies se taxonomizaron como dos genotipos de Bacteroides intermedius (entre 1983 y 1990) y de Prevotella intermedia (entre 1990 y 1992); sin embargo, hoy se reconocen como dos especies genéticamente distintas dentro del grupo Prevotella intermedia. En la actualidad son conocidas como "bacterias pigmentadas negras", ya que forman colonias brillantes y lisas de color gris, marrón claro o negro en la placa de agar sangre. Tienen una capacidad limitada para fermentar aminoácidos, y requieren de hemina y menadiona para crecer. Muchos taxones de Prevotella de la cavidad oral son potenciales patógenos oportunistas $(7,8)$.

La Prevotella intermedia ha sido reclasificada como Prevotella intermedia y Prevotella nigrescens. Ambas especies tienen caracteres fenotípicos similares entre sí; como el requisito de necesitar hierro para su crecimiento y la adquisición de la virulencia. La hemoglobina se encuentra en el líquido crevicular de los sitios de la EP y posiblemente podría ser una fuente de hierro para la Prevotella intermedia y nigrescens (9). Ambas son bacilos anaerobios gramnegativos, miembros del complejo naranja, que se encuentran entre las especies más encontradas de la placa subgingival (8); y, mientras la Prevotella intermedia es un miembro asociado a la periodontitis, la Prevotella nigrescens se ha detectado en la mayoría de los sujetos y no cambia en proporción de salud a enfermedad, debido a que desde inicio de la gingivitis natural, salud gingival y después de 14 días de gingivitis experimental, la Prevotella nigrescens es el microorganismo predominante. La Prevotella nigrescens produce polisacáridos que desempeñan un papel importante en el desarrollo de lesiones inflamatorias crónicas, mientras que la Prevotella intermedia aumenta la actividad de enzimas degradantes en la progresión de la periodontitis (4). Ambas producen una fluorescencia roja en la placa dental, que se reconoce para la clasificación de anaerobios pigmentados de negro, como el género de Porphyromonas (9).

Una dificultad para controlar a la Prevotella intermedia es que la bacteria tiene resistencia a muchos antibióticos, incluidos penicilinas, cefalosporinas y tetraciclinas; gracias a la formación de biopelículas que las hacen más resistentes $(10,11)$.

\section{EP en la paciente embarazada}

La EP actúa como factor de riesgo para el parto prematuro al aumentar las respuestas inflamatorias locales y sistémicas. Es así que la elevación de las hormonas esteroideas durante el embarazo pueden modificar la respuesta inflamatoria, generando una inflamación mayor a la esperada, en respuesta a niveles pequeños de placa (12).

La causa por la cual las hormonas sexuales femeninas afectan el periodonto durante el embarazo es variada. La encía contiene receptores de estrógeno y progesterona, de ahí que un incremento plasmático conduce a un aumento y acumulación; produciendo cambios vasculares, celulares, microbiológicos e inmunológicos (13). 
Del mismo modo, Markou et al. (14) mencionan que estas hormonas pueden influir en la proliferación celular y en la diferenciación y crecimiento de los queratinocitos y fibroblastos; dado que el estrógeno es el principal responsable de las alteraciones en los vasos sanguíneos y la progesterona estimula la producción de mediadores inflamatorios. Además, algunos microorganismos encontrados en la boca sintetizan enzimas para la síntesis de esteroides y su catabolismo, contribuyendo indirectamente a la EP.

Durante el embarazo, también se producen cambios en la fisiología y el metabolismo materno; por ejemplo, la resistencia vascular periférica disminuye para compensar los aumentos de renina y angiotensina. Este efecto se atribuye a las prostaglandinas específicas $\left(\mathrm{PGE}_{2}\right.$ y $\left.\mathrm{PGI}_{2}\right)$. En un embarazo normal, estas prostaglandinas se sintetizan en las membranas fetales: la decidua, el miometrio y la placenta. La producción de estas sustancias es proporcional a la edad gestacional, de modo que hay más prostaglandinas al final del embarazo que durante el primer trimestre. Un aumento local de mediadores pro inflamatorios puede interrumpir este delicado equilibrio; por lo tanto, una respuesta inflamatoria estimulada por una infección local (por ejemplo, EP) bajo este mecanismo contribuiría a la ruptura prematura de las membranas y la contracción uterina, lo que desencadenaría el parto prematuro o el aborto espontáneo (15). En este sentido, Iheozor-Ejiofor et al. (16) evaluaron los efectos del tratamiento de la EP en embarazadas para prevenir la morbilidad - mortalidad perinatal y materna, encontrando que no está claro si el tratamiento periodontal tiene un impacto en el parto prematuro y ayuda prevenir resultados adversos.

\section{Prevotella intermedia en gestantes con EP}

La etiología de la gingivitis durante el embarazo implica cambios en las biopelículas subgingivales o supragingivales y la influencia de las hormonas sexuales en el periodonto; demostrándose que hay asociaciones entre niveles elevados de Prevotella intermedia y los niveles de hormonas sexuales femeninas, dado que la Prevotella utiliza las hormonas como fuente de nutrientes. Los cambios cuantitativos en la biopelícula se dan debido al crecimiento excesivo de Prevotella intermedia, y están implicados en el aumento de la gravedad de la gingivitis durante el embarazo. Por lo que se debe tener en cuenta que los niveles de estrógeno y progesterona aumentan hasta el octavo mes del embarazo y desempeñan un papel importante en el aumento de la gravedad de la gingivitis (17 - 19).

Los cambios hormonales parecen estar correlacionados con el crecimiento de ciertas bacterias anaerobias gramnegativas, estimulando particularmente el crecimiento de la Prevotella intermedia y de Campylobacter rectus y Prevotella nigrescens (20).

El complejo naranja precede y crea condiciones para la retención del complejo rojo, que está compuesto por especies de Porphyromonas gingivalis, Treponema denticola y Tannerella forsythia. El complejo rojo es el agente etiológico de la EP crónica y está relacionado con un aumento en la profundidad de la bolsa y la presencia de sangrado durante el sondaje (6).

Durante la gestación las bacterias orales con capacidad invasiva, como la Prevotella intermedia y la Porphyromonas gingivalis, pueden translocarse al torrente sanguíneo y causar inflamación en sitios distantes, asociando a la EP con resultados adversos del nacimiento (1).

Tellapragada et al. (21) encontraron altas tasas de detección de Porphyromonas gingivalis (56\%), Prevotella nigrescens (44\%), Treponema denticola (32\%) y Prevotella intermedia (24\%) en embarazadas, junto con una asociación significativa con la EP ( $P<0.05$ ); donde las tasas de detección más altas de miembros del "complejo naranja" (Prevotella intermedius y Prevotella nigrescens) junto con $P$. gingivalis y Treponema denticola (complejo rojo) indican una coexistencia de miembros del complejo naranja y rojo. Borgo et al. (22) determinaron las condiciones gingivales y la detección cuantitativa de bacterias en mujeres embarazadas durante el segundo y tercer trimestre, y mujeres no embarazadas. Observaron que las embarazadas son más susceptibles a la gingivitis y que la Prevotella intermedia se hallaba en niveles más altos que otras bacterias. De forma similar, Usin et al. (23) concluyeron que la presencia de Prevotella intermedia y el $A$. actinomycetemcomitans en bolsas periodontales de embarazadas con diferente estado periodontal se asociaría con el parto prematuro y/o bajo peso al nacer.

Jelihovschi et al. (1) mencionan que en el embarazo las variaciones hormonales son condiciones favorables para desarrollar o exacerbar la periodontitis; donde la Prevotella intermedia se detecta con mayor frecuencia y en mayor abundancia en pacientes embarazadas. Coincidiendo con los resultados de Machado et al. (20), quienes también encontraron los recuentos más altos de Prevotella intermedia en las embarazadas; sin embargo, los patrones de colonización de las diferentes bacterias asociadas con la EP no fueron diferentes en la placa subgingival de mujeres embarazadas y no embarazadas.

Así mismo, Parthiban et al. (24) encontraron en la placa subgingival de mujeres preeclampsicas, frecuencias significativamente más altas de Prevotella intermedia. En las muestras de la placenta se descubrió que $P$. gingivalis, Prevotella intermedia y la expresión de los receptores Toll-like (TLR-4) y el factor nuclear de fase 
tardía $\kappa \mathrm{B}(\mathrm{NF}-\kappa \mathrm{B})$ tenían niveles significativamente más altos que en las mujeres embarazadas normotensivas. Concluyendo que existe una asociación entre la $P$. gingivalis y la Prevotella intermedia en la placenta de mujeres preeclampsicas con periodontitis. En este sentido, Blanc et al. (25) muestran que las bacterias orales pueden estar presentes en la placenta.

\section{Conclusión}

Se encuentra que sí hay relación entre la Prevotella intermedia con la presencia y la magnificación de las enfermedades periodontales durante la etapa del embarazo. Por lo cual, es siempre recomendable realizar la etapa preventiva y no quirúrgica del tratamiento periodontal en las mujeres embarazadas.

\section{Referencias bibliográficas}

1. Jelihovschi I, Dorneanu S, Serban R, Badescu A, Ursu R, Martu A, et al. Increased Detection Rate of Prevotella Intermedia among Pregnant Periodontitis Patients in Romania. J Biomed Sci \& Tech Res. 2018; 2(1), :2150-2154.

2. Kinane DF, Stathopoulou PG, Papapanou PN. Periodontal diseases. Nat Rev Dis Primers. 2017.

3. Socransky SS. Haffajee AD. Cugini MA, Smith C, Kent Jr. RL: Microbial complexes in subgingival plaque. J Clin Periodontol 1998; 25 : 134-144.

4. Zhang Y, Zhen M, Zhan Y, Song Y, Zhang Q, Wang J. Population-Genomic Insights into Variation in Prevotella intermedia and Prevotella nigrescens Isolates and Its Association with Periodontal Disease. Front Cell Infect Microbiol. 2017 Sep 21;7:409.

5. Canakci V, Canakci CF. Ildirim A, Ingec M, Eltas A, Erturk A. Periodontal disease increase the risk of severe pre-eclampsia among pregnant women. J Clin Periodontol 2007; 34:639-645.

6. Lima DP, Moimaz SA, Garbin CA, Sumida DH, Jardim EG Jr, Okamoto AC. Occurrence of Socransky Red Complex in Pregnant Women With and Without Periodontal Disease. Oral Health Prev Dent. 2015;13(2):169-176.

7. Ruan Y, Shen L, Zou Y, Qi Z, Yin J, Jian J, et al. Comparative genome analysis of Prevotella intermedia strain isolated from infected root canal reveals features related to pathogenicity and adaptation. BMC Genomics. 2015. $25 ; 16: 122$.

8. Rams TE, Sautter JD, Hsiao CY, van Winkelhogg AJ. Phenotypic identification of periodontal Prevotella intermedia/nigrescens group isolates validated by MALDI-TOF mass spectrometry. Anaerobe. $2018 ; 54: 201-204$.

9. Nomura Y, Takeuchi H, Okamoto M, Sogabe K, Okada A, Hanada N. Chair-side detection of
Prevotella Intermedia in mature dental plaque by its fluorescence. Photodiagnosis Photodyn Ther. 2017;18:335-341.

10. Jang EY, Kim M, Noh MH, Moon JH, Lee JY. .In Vitro Effects of Polyphosphate against Prevotella intermedia in Planktonic Phase and Biofilm. Antimicrob Agents Chemother. 2015; 60(2), 818-826.

11. Moon JH, Jang EY, Shim KS, Lee JY. In vitro effects of $\mathrm{N}$-acetyl cysteine alone and in combination with antibiotics on Prevotella intermedia. J Microbiol. 2015;53(5):321-329.

12. Chapple ILC, Mealey BL, Van Dyke TE, Bartold PM, Dommisch H, Eickholz P. Periodontal health and gingival diseases and conditions on an intact and a reduced periodontium: Consensus report of workgroup 1 of the 2017 World Workshop on the Classification of Periodontal and Peri-Implant Diseases and Conditions. J Periodontol. 2018;89 Suppl 1:S74S84.

13. Fajardo Puig ME, Rodríguez Reyes O, Rodríguez Bacallao A. MEDISAN. 2017;21(1):108-112.

14. Markou E, Boura E, Tsalikis L, Konstantinides A. The Influence of Sex Steroid Hormones on Gingiva of Women. The Open Dentistry Journal. 2009: 3, 114-119.

15. Latorre Uriza C, Velosa-Porras J, Roa NS, Quiñones Lara SM, Silva J, Ruiz AJ, Escobar Arregoces FM. Periodontal Disease, Inflammatory Cytokines, and PGE2 in Pregnant Patients at Risk of Preterm Delivery: A Pilot Study. Infect Dis Obstet Gynecol. 2018. $1 ; 2018: 7027683$.

16. Iheozor-Ejiofor Z, Middleton P, Esposito M, Glenny AM. Treating periodontal disease for preventing dverce birth outcomes in pregnant women. Cochrane Database Syst Rev. 2017 Jun 12;6:CD005297.

17. Usin MM, Tabares SM, Parodi RJ, Sembaj A. Periodontal conditions during the pregnancy associated with periodontal pathogens. J 
Investig Clin Dent. 2013; 4(1):54-59.

18. Novak MJ, Novak KF, Hodges JS, Kirakodu S, Govindaswami M, Diangelis A, et al. Periodontal Bacterial Profiles in Pregnant Women: Response to Treatment and Associations With Birth Outcomes in the Obstetrics and Periodontal Therapy (OPT) Study. J Periodontol. 2008 Oct;79(10):18701879.

19. Adriaens LM, Alessandri R, Spörri S, Lang NP, Persson GR.. Does Pregnancy Have an Impact on the Subgingival Microbiota?. J Periodontol. 2009; 80(1):72-81.

20. Machado FC, Cesar DE, Assis AV, Diniz CG, Ribeiro RA. Detection and enumeration of periodontopathogenic bacteria in subgingival biofilm of pregnant women. Braz Oral Res. 2012 Sep-Oct;26(5):443-449.

21. Tellapragada C, Eshwara VK, Acharya S, Bhat P, Kamath A, Vishwanath S, et al. Prevalence of Clinical Periodontitis and Putative Periodontal Pathogens among South Indian Pregnant Women Int J Microbiol. 2014;2014:420149.
22. Borgo PV, Rodrigues VA, Feitosa AC, Xavier KC, Avila-Campos MJ. Association between periodontal condition and subgingival microbiota in women during pregnancy: a longitudinal study. J Appl Oral Sci. 2014 NovDec;22(6):528-533.

23. Usin MM, Menso J, Rodríguez VI, González A, Tabares S, Parodi R. Association between maternal periodontitis and preterm and/or low birth weight infants in normal pregnancies. J Matern Fetal Neonatal Med. 2016;29(1):115119.

24. Parthiban PS, Mahendra J, Logaranjani A, Shanmugam S, Balakrishnan A, Junaid M, et al. Association between specific periodontal pathogens, Toll-like receptor-4, and nuclear factor- $\mathrm{\kappa B}$ expression in placental tissues of preeclamptic women with periodontitis . J Investig Clin Dent. 2018; 9(1), e12265.

25. Blanc V, O'Valle F, Pozo E, Puertas A, León R, Mesa F. Oral bacteria in placental tissues: increased molecular detection in pregnant periodontitis patients. Oral Dis. 2015 Oct;21(7):905-912.
Correspondencia:

artdent2000@hotmail.com
Fecha de recepción : 20 de marzo de 2020

Fecha de aceptación : 16 de mayo de 2020 